\title{
Characteristics of Dolutegravir and Bictegravir Plasma Protein Binding: A First Approach for the Study of Pharmacologic Sanctuaries
}

Thibaut Gelé ${ }^{1}$, Hélène Gouget ${ }^{2}$, Valérie Furlan ${ }^{1}$, Pierre-Hadrien Becker ${ }^{1}$, Anne-Marie Taburet $^{3}$, Olivier Lambotte ${ }^{1}$, and Aurélie Barrail-Tran ${ }^{1}$

${ }^{1} \mathrm{AP}-\mathrm{HP}$

${ }^{2} \mathrm{CEA}$

${ }^{3}$ INSERM

April 28, 2020

\begin{abstract}
Background and Purpose: The major obstacle to a functional cure for HIV infection is the persistence of a latent HIV reservoir. In addition, it is well recognized that the unbound concentration of a drug is considered the active moiety, which is available to cross cell membranes. Thus, this study aimed to characterize in vitro dolutegravir (DTG) and bictegravir (BIC) binding to either total human plasma proteins, human serum albumin (HSA) or human alpha-1-acid glycoprotein (HAAG). Experimental Approach: DTG and BIC protein binding was measured in vitro at a very wide range of concentrations, in either blank plasma or solutions containing HSA or HAAG. The pH effect was also studied on HSA binding at different pH. Bound and unbound drugs (DTG or BIC) were separated by ultrafiltration. Key Results: In human plasma, at therapeutic concentrations, the average DTG and BIC binding was $\sim 99.0 \%$. DTG and BIC had a preferential binding to HSA. DTG and BIC bound to two classes of albumin sites, one saturable with high affinity and one non-saturable with low affinity. A 0.2 -U decrease in $\mathrm{pH}$ led to a $2 \%$ decrease in DTG and BIC HSA binding. DTG bound to a non-saturable and low affinity HAAG. HAAG binding of BIC showed an atypical nonlinear binding. Conclusion and Implications: The two low affinity sites (on HSA and on HAAG) suggest that the high protein binding rate is not a limiting factor of the diffusion of DTG and BIC.
\end{abstract}

\section{Hosted file}

Gele_et al.doc available at https://authorea.com/users/309622/articles/440528-characteristicsof-dolutegravir-and-bictegravir-plasma-protein-binding-a-first-approach-for-the-study-ofpharmacologic-sanctuaries 